\title{
BMJ Open Cost-effectiveness of superficial femoral artery endovascular interventions in the UK and Germany: a modelling study
}

\author{
Benjamin C Kearns, ${ }^{1}$ Steven M Thomas ${ }^{2}$
}

To cite: Kearns BC,

Thomas SM. Cost-

effectiveness of superficial

femoral artery endovascular interventions in the UK and Germany: a modelling study. BMJ Open 2017;7:e013460. doi:10.1136/bmjopen-2016013460

- Prepublication history and additional material is available. To view please visit the journal (http://dx.doi.org/ 10.1136/bmjopen-2016013460).

Received 13 July 2016 Revised 15 September 2016 Accepted 3 October 2016

CrossMark

${ }^{1}$ The University of Sheffield, Sheffield, UK

${ }^{2}$ Sheffield Teaching Hospitals, Sheffield, UK

Correspondence to Mr Benjamin C Kearns; b.kearns@sheffield.ac.uk

\section{ABSTRACT}

Objectives: To assess the lifetime costs and costeffectiveness of 5 endovascular interventions to treat superficial femoral arterial disease.

Design: A model-based health economic evaluation. An existing decision analytical model was used, with updated effectiveness data taken from the literature, and updated costs based on purchasing prices.

Setting: UK and German healthcare perspectives were considered.

Participants: Patients with intermittent claudication of the femoropopliteal arteries eligible for endovascular treatment.

Methods: UK and German healthcare perspectives were considered, as were different strategies for reintervention.

Interventions: Percutaneous transluminal angioplasty (PTA) with bail-out bare metal stenting (assumed to represent the existing standard of care, and 4 alternatives: primary bare metal stents, drug-eluting stents, drug-eluting balloons (DEBs) and biomimetic stents).

Primary outcome measures: The incremental costeffectiveness ratio between 2 treatments, defined as the incremental costs divided by the incremental qualityadjusted life years (QALYs).

Results: Use of a biomimetic stent, BioMimics 3D, was always estimated to dominate the other interventions, having lower lifetime costs and greater effectiveness, as measured by QALYs. Of the remaining interventions, DEBs were always the most effective, and PTA the least effective. There was uncertainty in the cost-effectiveness results, with key drivers being the costs and effectiveness of the biomimetic stent along with the costs of DEBs.

Conclusions: All 4 of the alternatives to PTA were more effective, with the biomimetic stent being the most cost-effective. As there was uncertainty in the results, and all of the interventions have different mechanisms of action, all 4 may be considered to be alternatives to PTA.

\section{INTRODUCTION}

Worldwide, more than 200 million people are estimated to be living with peripheral
Strengths and limitations of this study

- This study is the first cost-effectiveness analysis to consider the four most promising alternatives to standard endovascular care for superficial femoral arterial disease: bare metal stents, drug-eluting stents, drug-eluting balloons (DEBs) and biomimetic stents.

- An existing decision analytical model, developed for a previous National Institute for Health Research funded economic evaluation, was adapted to include a new intervention (biomimetic stents) and updated with more recent evidence on costs and clinical effectiveness.

- We considered UK and German healthcare perspectives and were able to estimate the key drivers of decision uncertainty and the value of further research.

- The main limitations of the study are that there are no head-to-head comparisons between biomimetic stents and DEBs, the relatively small sample size for biomimetic stents and that heterogeneity in purchasing strategies led to variation in the estimated costs of the interventions.

arterial disease (PAD). ${ }^{1}$ The presence of PAD can have a significant impact on an individual's quality of life and on the costs incurred by the healthcare system, particularly if multiple interventions are required. ${ }^{2}$

Historically, percutaneous transluminal angioplasty (PTA) has been the main form of treatment for PAD. However, recent studies have demonstrated that alternative interventions such as bare metal stents (BMSs), drug-eluting stents (DESs) and drug-eluting balloons (DEBs) have been demonstrated to have favourable outcomes on clinically important measures such as long-term patency and the need for re-intervention. In addition to clinical effectiveness, it is also important to consider costeffectiveness, in other words do these interventions represent value for money? This is particularly important when considering interventions for $\mathrm{PAD}$, as these may be 
associated with an increased initial cost, but could result in savings over the longer term if the additional cost is offset by savings as a result of reduced re-interventions or lower complication rates. From a decision-making viewpoint, cost-effectiveness is important because not all effective interventions can be funded. Cost-effectiveness results provide a framework to assist debates about which interventions should and should not be funded.

A systematic review and economic evaluation of eight enhancements to PTA was conducted in May 2011. ${ }^{3}$ It identified that BMS, DES and DEB all resulted in an improvement in health outcomes for patients when compared to PTA alone. Of these interventions, the use of DEB was estimated to result in the lowest lifetime costs to the healthcare system and the greatest gains in health outcomes for patients. However, notable limitations of the economic evaluation included the lack of direct data on re-intervention rates for many of the interventions (instead changes in patency rates were assumed to reflect changes in re-intervention rates), and the limited follow-up data available, which was typically 12 months (6 months for DEB). Since the publication of the original economic evaluation, more results have been published which include evidence about the impact of interventions on re-intervention rates and longer follow-up data. In addition, evidence for a new class of swirling flow stents, known as biomimetic stents, has begun to emerge. ${ }^{4}$ This stent is differentiated from traditional BMSs as it imparts curvature to the artery, which increases the arterial wall shear stress, reducing the chance of restenosis.

The main aim of this study was to update the previous economic evaluation to incorporate the latest evidence relating to both the clinical effectiveness of treatments, along with their costs. A secondary aim was to assess the impact on cost-effectiveness of using different pricing schemes and different methods to treat re-intervention.

\section{METHODS}

\section{Previous economic evaluation}

The previous economic evaluation, funded by the National Institute for Health Research (NIHR), used a decision analytical model (DAM) to synthesise data on clinical effectiveness, costs to the healthcare system and patient's health-related quality of life. A comprehensive overview of the methods used to construct the DAM, and its input sources have previously been published. ${ }^{3} 5$ A brief overview of the key features of the model is presented here.

The patient population considered was those with symptomatic infrainguinal PAD suitable for endovascular treatment. It was assumed that PTA with bail-out BMS represented existing standard care. The eight alternative interventions considered were as follows: PTA with no with no bail-out stenting, PTA with bail-out DES, DEB, primary BMS, primary DES, endovascular brachytherapy, stent grafts and cryoplasty. For drug-eluting interventions, the drug considered was paclitaxel. It was assumed that all re-interventions performed were either bypass surgery (for acute failure; defined as within 30 days) or PTA with bail-out BMS (for failure after 30 days). A systematic review and meta-analysis were carried out to generate data on clinical effectiveness. ${ }^{6}$ Searches were conducted in May 2011. Six-month data from the Zilver PTX study were published after this review $^{7}$ but used within the DAM. Patency rates for standard care were taken from an existing meta-analysis. ${ }^{8}$

Patients entered the DAM on receiving PTA or one of the alternative interventions and had a probability of perioperative mortality or acute failure. For patients who remained alive and patent, long-term outcomes were modelled. These included late failure (which may or may not be accompanied by a return of symptoms), disease progression following failure, the development of contralateral symptoms or progressing to require amputation. Late failure accompanied by the return of symptoms led to re-intervention, which may also result in perioperative mortality. Patients could also die at any point.

Lifetime outcomes were considered, with costs reported in 2009-2010 British Pound Sterling and health outcomes measured using quality-adjusted life years (QALYs). QALYs are derived by applying a utility (weight) to life years experienced, with a utility of one indicating 'perfect health' and a utility of zero indicating a state equivalent to death. Utility data for PAD were taken from a previous economic evaluation. ${ }^{9}$ The perspective of the analysis was that of the UK National Health Service (NHS), with cost data taken from national guidance and national reference sources. For this study, additional analyses were performed which considered the perspective of the German healthcare system.

\section{Clinical effectiveness evidence}

Updated data on the clinical effectiveness of endovascular interventions were initially taken from the systematic review and meta-analysis of Jens et al. ${ }^{10}$ The searches for this review were conducted in November 2013. The measure of clinical effectiveness used for this study was the change in re-intervention rates, based on clinically driven target lesion revascularisation (TLR). Differences in TLR were reflected in the model by differences in the rate of re-intervention. For each intervention, relative risks (RRs) were calculated relative to PTA. For each intervention, there were no differences in rates of mortality or amputation.

In addition to the Jens et al meta-analysis, results for biomimetic stents were taken from the Mimics study. ${ }^{4}$ The Mimics study was a multicentre randomised controlled trial of patients receiving femoropopliteal intervention. A total of 76 patients were enrolled, of whom 50 received the BioMimics 3D stent (Veryan Medical, Horsham, UK), and 26 patients received a bare metal (nitinol) stent. Follow-up was for 2 years. As this was the only evidence about biomimetic stents that the authors 
were aware of, this study considered the costeffectiveness of the BioMimics 3D stent with the assumption that this was representative of the class of biomimetic stents. For DESs, only one publication ${ }^{7}$ was included in Jens et al. ${ }^{10}$ More recent data from this study (with longer follow-up) have been published. ${ }^{11}$ Hence for DESs this publication was used. Details on the derivation of RRs from the Jens $e t a l^{10}$ meta-analysis are provided in the online supplementary material.

For this study, it was assumed that the effects of interventions only lasted for up to 1 year after the point at which they were measured. As an example, if the effect of an intervention on TLR was measured at 2 years, it was assumed that this effect would persist for up to 3 years. This assumption was used to reflect the fact that different studies reported effectiveness data for differing lengths of follow-up. Where studies reported effectiveness data at multiple time-points, the longest follow-up time was used.

The use of DEB for re-interventions (instead of PTA) was also considered. ${ }^{12}$ Hence, two analyses were conducted from a UK perspective; one where PTA was used to treat re-interventions, the other where DEB was used. The analysis from a German perspective only considered the use of DEB. It was assumed that the effectiveness of DEB when used for re-interventions was the same as when used for the initial intervention.

Of the eight interventions included in the previous economic evaluation, the following were not considered for this study: cryoplasty, stent graft, endovascular brachytherapy, PTA with no bail-out stenting and PTA with bail-out DESs. This was because no data were available relating to the effects of these interventions on TLR rates.

\section{Evidence on costs}

Within the previous economic evaluation, cost data were primarily drawn from a cost-effectiveness analysis performed in support of guidance issued by the National Institute for Health and Care Excellence (NICE). ${ }^{13}$ However, there was large uncertainty in these costs. Hence for this study the costs of each intervention were updated. The costs of PTA with bail-out BMSs were taken from national NHS reference costs. ${ }^{14}$ It was assumed that $32.4 \%$ of operations would require bail-out stenting, based on values presented in the NICE costeffectiveness analysis. ${ }^{13}$ The costs of the remaining interventions were based on a mixture of company prices, hospital prices and expert opinion. It was assumed that PTA or DEB would cost the same regardless of whether it was the primary intervention or a re-intervention. A sensitivity analysis was conducted in which the cost of a re-intervention was triple that of the initial procedure.

For the analysis from a German perspective, the cost of PTA with bail-out bare metal stents was obtained by converting the UK pound sterling price to Euros, assuming a conversion rate of 1.2. Costs for the remaining interventions were supplied by German purchasing organisations. As there is no centrally agreed price for each intervention, three different sets of estimates were supplied. The cost-effectiveness of the interventions under each set of costs was assessed.

The interventions considered for this economic evaluation, along with the clinical and cost data used, are summarised in table 1.

The costs of on-going PAD management were also included in this study. It was assumed that patients with intermittent claudication would require one outpatient appointment every 3 months, while patients with critical limb ischaemia would require a monthly outpatient appointment and half-an-hour of a nurse's time once every 2 weeks. These assumptions were based on those used in the previous economic evaluation by Sculpher $e t a \ell^{9}$ and were costed using national reference sources. ${ }^{15}$ Details of on-going resource use following an amputation were not available, so an annual cost of £23 502 was used, taken from the NICE cost-effectiveness analysis. ${ }^{13}$

\section{Assessment of cost-effectiveness}

Interventions were compared in a fully incremental analysis for patients with intermittent claudication of the femoropopliteal arteries. The analysis took either the perspective of the UK NHS or the German healthcare system, along with personal social services. Estimates of costs to the healthcare system and benefits to patientsas measured by QALYs - were considered over a lifetime horizon. Costs were reported in either 2013-2014 British Pound Sterling or Euros. Costs and QALYs were discounted at a rate of $3.5 \% /$ year, as recommended by NICE. ${ }^{16}$ If an intervention generated more QALYs at less cost than another intervention, the former dominated the latter. Otherwise, the cost-effectiveness of an intervention is determined by the willingness of a decision maker to pay for an additional QALY. As this willingness to pay is typically unknown, a range of different values were considered. To generate results, 1000 probabilistic sensitivity analysis (PSA) runs were implemented. These willingness to pay values may be compared to incremental cost-effectiveness ratios (ICERs), defined as the incremental costs (when comparing two interventions) divided by their incremental effects.

Value of information analyses were performed using the Sheffield Accelerated Value of Information webbased application. ${ }^{17} 18$ The amount that should be spent on conducting further research (known as the expected value of perfect information) was estimated for England $^{19}$ and Germany, ${ }^{1}{ }^{20}$ assuming that the prevalence of PAD was 360599 and 1930000 , respectively, that $5.5 \%$ of patients with PAD would require an endovascular procedure (based on the 20000 procedures for in England for $2010 / 2011^{5}$ ) and that any intervention would be adopted for 10 years with a willingness to pay of $£ 20000$. The partial expected value of perfect information was also calculated to assess which parameters contributed the most to decision uncertainty. 
Table 1 Clinical and cost data used

\begin{tabular}{lllllll}
\hline & & & \multicolumn{3}{c}{ Cost estimates (2013/2014) } \\
\cline { 5 - 7 } Intervention & $\begin{array}{l}\text { RR; return of } \\
\text { symptoms* }\end{array}$ & $\begin{array}{l}\text { Time period } \\
\text { (months) }\end{array}$ & UK £ & $€$ (set 1) & $€($ set 2) & $€($ (set 3) \\
\hline PTA with bail-out bare metal stents & 1 & - & $£ 3248$ & $€ 3898$ & $€ 3898$ & $€ 3898$ \\
Bare metal stents & 0.7261 & 12 & $£ 3848$ & $€ 4354$ & $€ 4498$ & $€ 4282$ \\
Drug-eluting stents & 0.5216 & 60 & $£ 4208$ & $€ 4678$ & $€ 5053$ & $€ 5038$ \\
Drug-eluting balloons & 0.2739 & 18 & $£ 4604$ & $€ 4848$ & $€ 4888$ & $€ 5360$ \\
Biomimetic (BioMimics 3D) stent & 0.2711 & 24 & $£ 3968$ & $€ 4618$ & $€ 4618$ & $€ 4618$ \\
\hline
\end{tabular}

All data are from Jens et al, with the exception of the biomimetic BioMimics 3D stent, which comes from the Mimics trial, and drug-eluting stents, which comes from the 5-year Zilver PTX results.

*Defined as the need for clinically driven target lesion revascularisation.

PTA, percutaneous transluminal balloon angioplasty; RR, relative risk.

\section{RESULTS}

Average lifetime cost-effectiveness results from the 1000 PSA runs are presented in tables 2 and 3, in ascending order of costs. Table 2 presents results from a UK perspective under the two scenarios considered (re-interventions with either PTA or DEB) while table 3 presents results from a German perspective using three different sets of pricing estimates (all re-interventions were with DEB). In addition to lifetime costs and QALYs, table 2 also presents the lifetime costs of re-operations and PAD management is also displayed. These costs were similar when using a German perspective, so are not reported in table 3.

The results from all five analyses indicated that the use of the biomimetic BioMimics 3D stent was associated with the lowest lifetime costs and the highest number of QALYs. In other words, the BioMimics 3D stent dominated all of the other interventions. Of the remaining interventions, the use of PTA with bail-out BMSs (assumed current practice) was always associated with the lowest lifetime costs, but also the lowest number of QALYs. Of the remaining three interventions, the use of DEBs was always estimated to be the most effective (in terms of QALYs). For three of the five analyses considered, the use of DEBs was estimated to dominate the use of BMSs (being more effective and less costly). DESs were estimated to be cheaper than DEBs in three of the five analyses.

There were two main drivers for the cost savings observed for the use of biomimetic stents: a reduction in the number of repeat operations required, and a reduction in the average time spent with PAD (due to a loss of patency and return of symptoms). These costs are displayed in table 2. The use of any of the interventions was estimated to lead to a reduction in the average lifetime costs of re-operations and the average lifetime cost of PAD management, relative to the use of PTA with bail-out BMSs. The use of DEB for re-interventions instead of PTA lowered the lifetime costs of PAD management, but increased the costs of re-operations.

Results from the sensitivity analysis (using a UK perspective), which assumed that repeat procedures cost an average of $£ 9744$ (instead of $£ 3248$ ), indicated that the use of either the biomimetic BioMimics 3D stent, DEBs or DESs would result in lower lifetime costs than the use of PTA with bail-out BMSs. Of these interventions, the BioMimics 3Ds stent was still associated with the lowest lifetime costs, followed by the use of DEBs.

\begin{tabular}{|c|c|c|c|c|}
\hline Intervention & Costs $(£)$ & QALYs & Costs of re-operations $(£)$ & Costs of PAD management $(£)$ \\
\hline \multicolumn{5}{|l|}{ All re-interventions PTA } \\
\hline Biomimetic (BioMimics 3D) stents & $£ 10715$ & 6.302 & $£ 2893$ & $£ 524$ \\
\hline PTA with bail-out bare metal stents & $£ 11328$ & 6.213 & $£ 3454$ & $£ 1153$ \\
\hline Drug-eluting stents & $£ 11348$ & 6.275 & $£ 3047$ & $£ 713$ \\
\hline Drug-eluting balloons & $£ 11406$ & 6.300 & $£ 2920$ & $£ 540$ \\
\hline Bare metal stents & $£ 11498$ & 6.242 & $£ 3273$ & $£ 950$ \\
\hline \multicolumn{5}{|l|}{ All re-interventions DEB } \\
\hline Biomimetic (BioMimics 3D) stents & $£ 10838$ & 6.330 & $£ 3261$ & $£ 312$ \\
\hline PTA with bail-out bare metal stents & $£ 11019$ & 6.286 & $£ 3787$ & $£ 544$ \\
\hline Bare metal stents & $£ 11309$ & 6.302 & $£ 3612$ & $£ 457$ \\
\hline Drug-eluting stents & $£ 11325$ & 6.318 & $£ 3399$ & $£ 368$ \\
\hline Drug-eluting balloons & $£ 11515$ & 6.329 & $£ 3287$ & $£ 320$ \\
\hline
\end{tabular}


Table 3 Cost-effectiveness results; German perspective

\begin{tabular}{|c|c|c|c|c|}
\hline Intervention & Costs $(€)$ set 1 & Costs $(€)$ set 2 & Costs $(€)$ set 3 & QALYs \\
\hline Biomimetic (BioMimics 3D) stents & $€ 11576$ & $€ 11590$ & $€ 11764$ & 6.330 \\
\hline PTA with bail-out bare metal stents & $€ 11770$ & $€ 11786$ & $€ 11984$ & 6.286 \\
\hline Drug-eluting balloons & $€ 11848$ & $€ 11902$ & $€ 12549$ & 6.329 \\
\hline Drug-eluting stents & $€ 11886$ & $€ 12276$ & $€ 12439$ & 6.318 \\
\hline Bare metal stents & $€ 11911$ & $€ 12071$ & $€ 12044$ & 6.302 \\
\hline
\end{tabular}

Values are lifetime averages per patient. All re-interventions are with drug-eluting balloons.

PTA, percutaneous transluminal balloon angioplasty; QALYs, quality-adjusted life years.

The model results suggested that, while the use of the biomimetic BioMimics 3D stent dominated each of the other interventions, there was some uncertainty in these results. This uncertainty is reflected in figure 1 , which displays the incremental costs and effects estimated from each PSA run for each of the interventions compared to of PTA with bail-out BMSs (under a UK perspective, with PTA for re-interventions). The cloud of overlapping points highlights the variation in cost-effectiveness results. In particular, there was little estimated difference in the effectiveness of the BioMimics 3D stent and DEBs, with a difference in average lifetime QALYs of 0.002. Over the 1000 PSA runs, the use of the BioMimics 3D stent was estimated to dominate use of DEBs $39.2 \%$ of the time, but be dominated $16.9 \%$ of the time.

The probability that each of the interventions was the most cost-effective is displayed in a cost-effectiveness acceptability curve in figure 2 (under a UK perspective, with PTA for re-interventions). At a willingness to pay of $£ 0$ (which implies that QALY gains are not considered), the probability that the biomimetic BioMimics 3D stent was cost-effective was $39.2 \%$, while for DEBs and DESs the probabilities were $15.7 \%$ and $16.0 \%$, respectively. As willingness to pay values increased, these values changed to about 59\% (BioMimics 3D stent), 31\% (DEBs) and $8 \%$ (DESts)

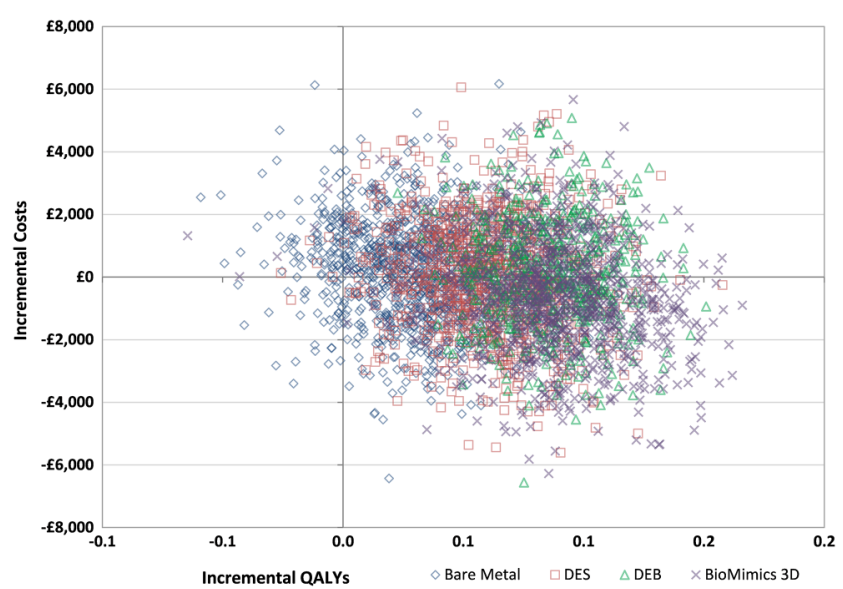

Figure 1 Scatterplot of incremental costs and QALYs for each intervention, relative to standard care. DEB, drug-eluting balloon; DES, drug-eluting stent; QALYs, quality-adjusted life years.
The maximum amount that it is worth spending on further research was estimated to be about $£ 136$ million for England and about $€ 1.1$ billion for Germany. The main sources of decision uncertainty were the costs of a biomimetic stent and DEBs, which together contributed to $68 \%$ of the decision uncertainty. In comparison, the contribution to decision uncertainty due to the cost of DESs was $11 \%$ while for BMSs it was $1 \%$. Uncertainty in the effectiveness of the biomimetic BioMimics 3D stent contributed to $11 \%$ of the decision uncertainty; all other inputs contributed $<1 \%$ each.

\section{DISCUSSION}

The cost-effectiveness results suggested that the use of the biomimetic BioMimics 3D stent dominated all of the other interventions by being cheaper and more effective over a patient's lifetime. However, there was some uncertainty in these results. All of the interventions considered were estimated to be more effective than the use of PTA with bail-out BMSs, and relative to this their ICERs were all well below the value of $£ 20000$ stated as being a threshold for cost-effectiveness in recent NICE guidance. $^{16}$

Within the UK, NICE make decisions about which interventions should be made available via the NHS.

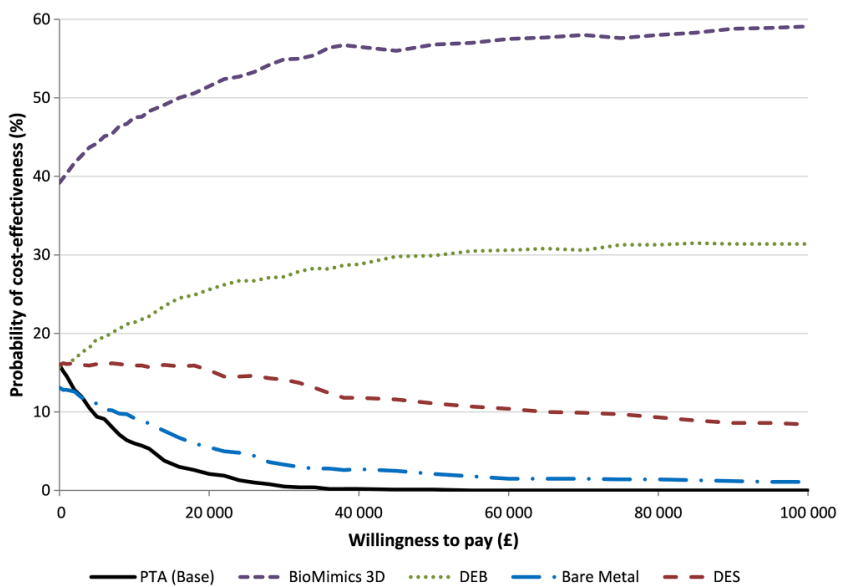

Figure 2 The probability that each of the interventions is cost-effective, for different willingness to pay values. DEB, drug-eluting balloon; DES, drug-eluting stent; QALYs, quality-adjusted life years. 
Cost-effectiveness data have been identified as a key component in NICE decision-making. ${ }^{21}$ The results presented here suggest that any of the alternatives to PTA are potentially cost-effective options, with the use of biomimetic stents predicted to lead to cost savings.

The use of the biomimetic stents has not been considered in previous economic evaluations, due to a lack of evidence. Of the other interventions considered in the NIHR-funded evaluation, ${ }^{5}$ results and their interpretation were similar to this evaluation with the exception of the lifetime costs for DEBs. In the previous evaluation, these were estimated to be cheaper than the lifetime costs for PTA with bail-out BMSs, but for this evaluation they were estimated to be $£ 691$ more expensive. The key driver for this is the different method for estimating the costs of an operation with a DEB. In the previous evaluation, this was estimated to cost $£ 232$ more than PTA with bail-out BMSs, with improved costing information for this evaluation the cost was £1356 more. Hence, the previously used value of $£ 232$ is likely to be an underestimate. However, the use of DEBs was still estimated to have a higher probability of being cost-effective compared to PTA with bail-out BMSs for all willingness to pay values. The economic evaluation performed to support NICE guidance on PAD did not consider either of the drug-eluting technologies due to a lack of evidence over revascularisation rates. ${ }^{11}$ These data are now available and have been incorporated into this model to suggest that drug-eluting alternatives to PTA may be cost-effective.

There are limitations with this work. First, there is a lack of head-to-head comparisons between some of the interventions; in particular, there is no direct comparison between the effectiveness of biomimetic stents and DEBs. Second, differences in purchasing strategies result in variation in the costs for the interventions considered. The impact of this variation is highlighted by the value of information results, which show that $68 \%$ of the decision uncertainty is driven by variation in the costs of biomimetic stents and DEBs. Third, the cost-effectiveness results reported for this study were from a model-based economic evaluation. The use of a model was necessary to synthesise data on effectiveness, costs and health-related quality of life from multiple sources of evidence. However, models are by definition a simplification of reality ${ }^{22}$ and require a number of assumptions regarding the clinical pathway modelled and the derivation of lifetime costs. The assumptions used for this study have been described in detail here and previously. ${ }^{3}$ Despite this, there is the potential that one or more of these assumptions may be incorrect or that they may not be applicable in different healthcare systems, potentially limiting the generalisability of these results. Finally, there is uncertainty in the results due to the relatively small sample size of the MIMCS study when compared to the other interventions. This uncertainty is captured within the PSA as reflected, for example, by the overlapping cloud of points for figure 2. Decisions are usually made in a situation of uncertainty, and the results from economic evaluations can assist in the decision-making process. However, there may be reluctance to choose the most cost-effective intervention (the BioMimics 3D stent) due to the relatively small evidence base. Hence, further research into this intervention may have merit.

The maximum amount that should be spent on further research was calculated using expected value of information techniques. The estimated value for Germany was about $€ 1.1$ billion, driven by the scale of disease in this country. ${ }^{23}$ This value is an approximation as it assumes that all of the decision uncertainty can be reduced by collecting more data. However, it emphasises the fact that future research into this disease is likely to be a cost-effective use of resources in Germany.

Since the previous economic evaluation, ${ }^{5}$ there have been two further studies reporting on the costeffectiveness of endovascular interventions for femoropopliteal arterial disease. One study ${ }^{24}$ considered the 24-month economic impact of DEB, DES and BMS relative to PTA. German and US healthcare perspectives were considered. The authors found that, based on pooled TLR data, all three alternatives were more effective than PTA, with both of the drug-eluting interventions also having lower overall costs. An extension to the work considered the same outcomes, but with a UK perspective, ${ }^{25}$ with device costs from market research data. The authors found that while all of the alternatives to PTA were again more clinically effective, they were also all more expensive. Neither study considered lifetime outcomes. In addition, a key assumption of both studies was that TLR rates for PTA could be pooled across all studies. The justification for this assumption of a homogenous patient population is unclear, as there is substantial variation in PTA TLR rates. For example, pooling BMS studies give a 12-month TLR for PTA of $19.3 \%$, while pooling DEB studies give a 6-month TLR for PTA of $31 \%^{10}$ (12-month data not available).

This study looked at four alternative interventions to the use of PTA. Each of these interventions was associated with an increased cost for the initial operation, but also with a decrease in subsequent costs for re-operations and the management of PAD. On purely cost-effectiveness grounds, the use of the biomimetic BioMimics 3D stent is recommended, as it was estimated to be more effective and less costly than any of the other interventions considered. However, each of the interventions has a slightly different mechanism of action by which it delays the need for revascularisation. Given the importance of tailoring treatment to the individual, and the uncertainty in the presented cost-effectiveness results, all four interventions may be considered to be potential alternatives to the use of PTA.

Impact on daily practice

In settings where there are restricted healthcare budgets, it is becoming increasingly important to 
consider not just the effectiveness of interventions, but also their cost-effectiveness. The use of alternative interventions to angioplasty, in particular biomimetic stents or DEBs, is likely to be cost-effective. The use of a biomimetic stent appears to be the most effective and also the cheapest over a patient's lifetime, although there is uncertainty in this conclusion.

Contributors BCK and SMT involved in the design of the study. BCK performed the health economic evaluation and the first draft of the manuscript, which was critically revised by SMT.

Disclaimer The views expressed are those of the authors and not necessarily those of Veryan Medical Ltd. The funders of this study had no role in the study design, data collection, data analysis, data interpretation or writing of the report.

Funding This work was funded by Veryan Medical Ltd, Horsham, UK.

Competing interests BCK has received speaking honoraria from Medtronic and Veryan.

Provenance and peer review Not commissioned; externally peer reviewed.

Data sharing statement No additional data are available.

Open Access This is an Open Access article distributed in accordance with the Creative Commons Attribution Non Commercial (CC BY-NC 4.0) license, which permits others to distribute, remix, adapt, build upon this work noncommercially, and license their derivative works on different terms, provided the original work is properly cited and the use is non-commercial. See: http:// creativecommons.org/licenses/by-nc/4.0/

\section{REFERENCES}

1. Fowkes FG, Rudan D, Rudan I, et al. Comparison of global estimates of prevalence and risk factors for peripheral artery disease in 2000 and 2010: a systematic review and analysis. Lancet 2013;382:1329-40.

2. Burket MW. Restenosis and the New Health Care Economy. Endovascular Today 2013;12:42-8.

3. Simpson EL, Kearns B, Stevenson MD, et al. Enhancements to angioplasty for peripheral arterial occlusive disease: systematic review, cost-effectiveness assessment and expected value of information analysis. Health Technol Assess 2014;18:1-252.

4. Zeller T, Gaines PA, Ansel GM, et al. A helical centerline stent improves patency: two-year results from the randomized Mimics trial. Circ Cardiovasc Interv 2016;9:pii:e002930.

5. Kearns BC, Michaels JA, Stevenson MD, et al. Cost-effectiveness analysis of enhancements to angioplasty for infrainguinal arterial disease. Br J Surg 2013;100:1180-8.

6. Simpson EL, Michaels JA, Thomas SM, et al. Systematic review and meta-analysis of additional technologies to enhance angioplasty for infrainguinal peripheral arterial occlusive disease. $\mathrm{Br} J$ Surg 2013;100:1128-37.

7. Dake MD, Ansel GM, Jaff MR, et al. Zilver PTX Investigators. Paclitaxel-eluting stents show superiority to balloon angioplasty and bare metal stents in femoropopliteal disease twelve-month zilver
PTX randomized study results. Circ Cardiovasc Interv 2011;4:495-504.

8. Hunink MG, Wong JB, Donaldson MC, et al. Patency results of percutaneous and surgical revascularization for femoropopliteal arterial disease. Med Decis Making 1994;14:71-81.

9. Sculpher M, Michaels J, McKenna M, et al. A cost-utility analysis of laser-assisted angioplasty for peripheral arterial occlusions. Int J Technol Assess Health Care 1996;12:104-25.

10. Jens S, Conijn AP, Koelemay MJ, et al. Randomized trials for endovascular treatment of infrainguinal arterial disease: systematic review and meta-analysis (part 1: above the knee). Eur J Vasc Endovasc Surg 2014;47:524-35.

11. Dake MD, Ansel GM, Jaff MR, et al. Zilver PTX Investigators. Durable clinical effectiveness with paclitaxel-eluting stents in the femoropopliteal artery 5-year results of the Zilver PTX Randomized trial. Circulation 2016;133:1472-83; discussion 1483.

12. Virga V, Stabile E, Biamino G, et al. Drug-eluting balloons for the treatment of the superficial femoral artery in-stent restenosis: 2-year follow-up. JACC Cardiovasc Interv 2014;7:411-15.

13. National Institute for Health and Care Excellence. Lower limb peripheral arterial disease: diagnosis and management. Clinical Guideline 147. https://www.nice.org.uk/guidance/cg147 (accessed Sep 2016).

14. Department of Health. 2013-14 reference costs publication. https:// www.gov.uk/government/publications/

nhs-reference-costs-2013-to-2014 (accessed Sep 2016).

15. Curtis L. PSSRU Unit costs of health \& social care, 2013. University of Kent (United Kingdom) 2013. http://www.pssru.ac.uk/ project-pages/unit-costs/2013/ (accessed Sep 2016).

16. National Institute for Health and Care Excellence. Guide to the methods of technology appraisal 2013. https://www.nice.org.uk/ process/pmg9/chapter/foreword (accessed Sep 2016).

17. Strong M, Oakley JE, Brennan A. Estimating multiparameter partial expected value of perfect information from a probabilistic sensitivity analysis sample: a nonparametric regression approach. Med Decis Making 2014;34:311-26.

18. The University of Sheffield. Sheffield accelerated value of information. http://savi.shef.ac.uk/SAVI/ (accessed Sep 2016).

19. Health and Social Care Information Centre. Quality and outcomes framework-2013-14. http://digital.nhs.uk/catalogue/PUB15751 (accessed Sep 2016).

20. Kröger K, Stang A, Kondratieva J, et al. Heinz Nixdorf Recall Study Group. Prevalence of peripheral arterial diseaseresults of the Heinz Nixdorf recall study. Eur J Epidemiol 2006;21:279-85.

21. Dakin H, Devlin N, Feng $Y$, et al. The influence of cost-effectiveness and other factors on NICE decisions. Health Econ 2014;24:1256-71.

22. Caro JJ, Briggs AH, Siebert U, et al. ISPOR-SMDM Modeling Good Research Practices Task Force. Modeling good research practicesoverview: a report of the ISPOR-SMDM modeling good research practices task force-1. Med Decis Making 2012;32:667-77.

23. Hirsch AT, Duval S. The global pandemic of peripheral artery disease. Lancet 2013;382:1312-14.

24. Pietzsch JB, Geisler BP, Garner AM, et al. Economic analysis of endovascular interventions for femoropopliteal arterial disease: a systematic review and budget impact model for the United States and Germany. Catheter Cardiovasc Interv 2014;84:546-54.

25. Katsanos K, Geisler BP, Garner AM, et al. Economic analysis of endovascular drug-eluting treatments for femoropopliteal artery disease in the UK. BMJ Open 2016;6:e011245. 